\title{
Endoscopic brush cytology: a screening and surveillance tool for esophageal malignancy.
}

\author{
Dr. Kajal Salpekar ${ }^{1}$, Dr. Sadhana Mahore ${ }^{2}$, Dr. Pragati Karmarkar ${ }^{3}$. \\ ${ }^{1}$ Resident Pathology, ${ }^{2}$ Professor and Head, ${ }^{3}$ Associate Professor, Department of Pathology, NKP Salve \\ Institute of Medical Sciences, Nagpur 440019, Maharashtra, India
}

\begin{abstract}
Upper gastrointestinal tract is a common site for various lesions, especially malignant tumors. In India, esophageal and gastric cancers are the most common cancers found in men, while esophageal cancer ranks third among women after the carcinoma of breast and cervix. Squamous cell carcinoma is still the most common esophageal malignancy in India. Adenocarcinoma presents a decade earlier than squamous cell carcinoma.

Esophageal cancer is an aggressive disease with a generally poor prognosis and most of the malignancies are diagnosed in the advanced stage of the disease. Esophageal malignancy most likely develops through a dysplasia-neoplasia sequence similar to other forms of cancer. High grade squamous cell dysplasia and Barrett's esophagus are the two established premalignant conditions of squamous cell carcinoma and adenocarcinoma respectively. It was observed that the age of presentation of premalignant lesions was earlier than their malignant counterpart.

The endoscopic brush cytology plays an important role in the diagnosis of premalignant and malignant esophageal lesions. Through endoscopic brush, cytological samples can be taken from the precise suspected lesion.

The aim of the study was to evaluate premalignant and malignant esophageal lesions using endoscopic brush cytology in patients presenting with complaints pertaining to upper gastrointestinal tract.
\end{abstract}

Keywords: Barrette's, Brush Cytology, Endoscopy, Esophagus.

\section{INTRODUCTION:}

Upper gastrointestinal tract is a common site for various lesions, especially malignant tumors. In India, esophageal and gastric cancers are the most common cancers found in men, while esophageal cancer ranks third among women after the carcinoma of breast and cervix. Esophageal cancer is an aggressive disease with a generally poor prognosis. It is the sixth frequent site among all the cancers worldwide \& $80 \%$ cases are seen in developing countries. The prevalence of esophageal cancer has increased six times in the recent three decades, which was the most rapid increase between major malignancies [1].

Early esophageal malignancies are asymptomatic and highly curable. Unfortunately, most of the malignancies are diagnosed in the advanced stage of the disease. The 5-year survival rate of early esophageal cancer is $83.5 \%$ [2].

As in most malignant diseases, the prognosis and survival of patients with esophageal malignancy mostly depend on the extent of spread of the disease process when the disease was diagnosed. Therefore, early detection of malignancy can improve therapeutic results and reduces morbidity and mortality.

Endoscopy is an outpatient procedure and is considered minimally invasive and does not require any significant recovery after the procedure. The endoscopic visualization plays an important role in differential diagnosis of esophageal lesion [3]. Through endoscopic brush, cytological samples can be taken from the precise suspected lesion. The technique of brush cytology through endoscope retrieves epithelial cells from a large surface area of mucosa. As malignant cells posses a lower level of intercellular cohesion than normal cells, brushing can selectively sample these dyshesive cells. This procedure is non-invasive, cost-effective and has a rapid turn-over time [4].

This study was done to evaluate premalignant and malignant esophageal lesions using endoscopic brush cytology in patients presenting with complaints pertaining to upper gastrointestinal tract. The ultimate goal was to detect neoplasia or lesions at risk of developing neoplasia, allowing intervention or surveillance that leads to improved outcomes such as a reduced incidence of cancer or cancer deaths [5].

\section{MATERIAL AND MeTHODS:}

The study was a hospital based cross -sectional study, done on outdoor and admitted patients during a period of 2 years from 2011 to 2013 at tertiary care institute. Total of 105 patients, both male and female, of all age group with the specified complaints were evaluated in the study. 
Patients having upper gastrointestinal complaints like nausea, vomiting, Retrosternal burning pain, regurgitation, dysphagia, anorexia, weight loss, upper gastrointestinal bleeding, tobacco chewing, smoking and chronic alcohol intake ; were subjected to upper gastrointestinal endoscopic brush cytology.

The endoscopy was done using fiberoptic video endoscope (Olympus CV-150 series). After visual examination of the lesion, a cytological brush made up of small nylon bristles at the tip was passed through the instrument channel. The head of the brush was advanced out of its sleeve and rubbed and rolled repeatedly across the surface of the lesion; a circumferential sweep of the margin and base of an ulcer is desirable. The brush was then pulled back into the sleeve, and both were withdrawn together. The brush was protruded, wiped over glass slides and then the slides were rapidly fixed in $95 \%$ ethyl alcohol before air-drying and kept for a minimum of 30 minutes for fixation. These slides were stained by Haematoxylin \& Eosin (H\&E) and Papanicoloau stain.

\section{RESULT:}

Out of 105 patients evaluated 16 were found to have premalignant and 19 had malignant lesions. Seven cases of high grade squamous cell dysplasia (HGSD) and nine Barrett's esophagus (BE) constituted the premalignant lesions. Among the malignant lesions 17 patients had squamous cell carcinoma (SCC) while 2 patients had adenocarcinoma (ADC). Rest of the patients had normal or non-neoplastic cytology.

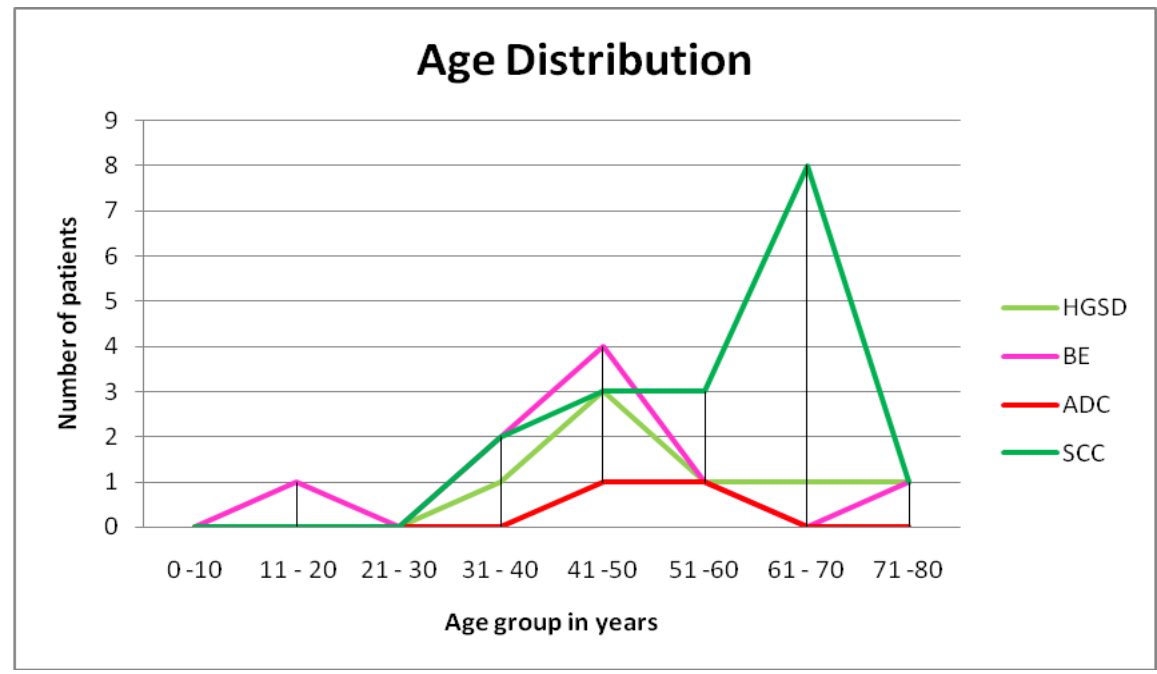

SCC- Squamous Cell Carcinoma, ADC-Adenocarcinoma, HGSD - high grade squamous cell dysplasia, BE - Barrett esophagus

Fig. 1: Age wise distribution of premalignant and malignant esophageal lesions

Premalignant lesion like HGSD was more common in male in the fifth decade and BE in female in fourth decade. Among the malignant lesions SCC was more common in male in $7^{\text {th }}$ decade while ADC in male $6^{\text {th }}$ decade (Fig.1).

Table 1: Age wise distribution of the premalignant and malignant esophageal lesions.

\begin{tabular}{|c|c|c|c|c|c|c|c|c|}
\hline$\underbrace{\text { Age (yrs) }}_{\text {Lesions }}$ & 0 to 10 & 11 to 20 & 21 to 30 & 31 to 40 & 41 to 50 & 51 to 60 & 61 to 70 & 71 to 80 \\
\hline HGSD & 0 & 0 & 0 & 1 & 3 & 1 & 1 & 1 \\
\hline $\mathbf{B E}$ & 0 & 1 & 0 & 2 & 4 & 1 & 0 & 1 \\
\hline$\overline{\mathrm{ADC}}$ & 0 & 0 & 0 & 0 & 1 & 1 & 0 & 0 \\
\hline SCC & 0 & 0 & 0 & 2 & 3 & 3 & 8 & 1 \\
\hline
\end{tabular}

Thus, it was observed that the premalignant lesions (HGSD \& BE) usually presented a decade or two earlier than their respective malignant counterpart. (Table 1)

Patients with Barrett's esophagus had history of retrosternal / epigstric burning pain and regurgitation. HGSD, squamous cell carcinoma and adenocarcinoma presented with dysphagia as a most common presenting complaint. 


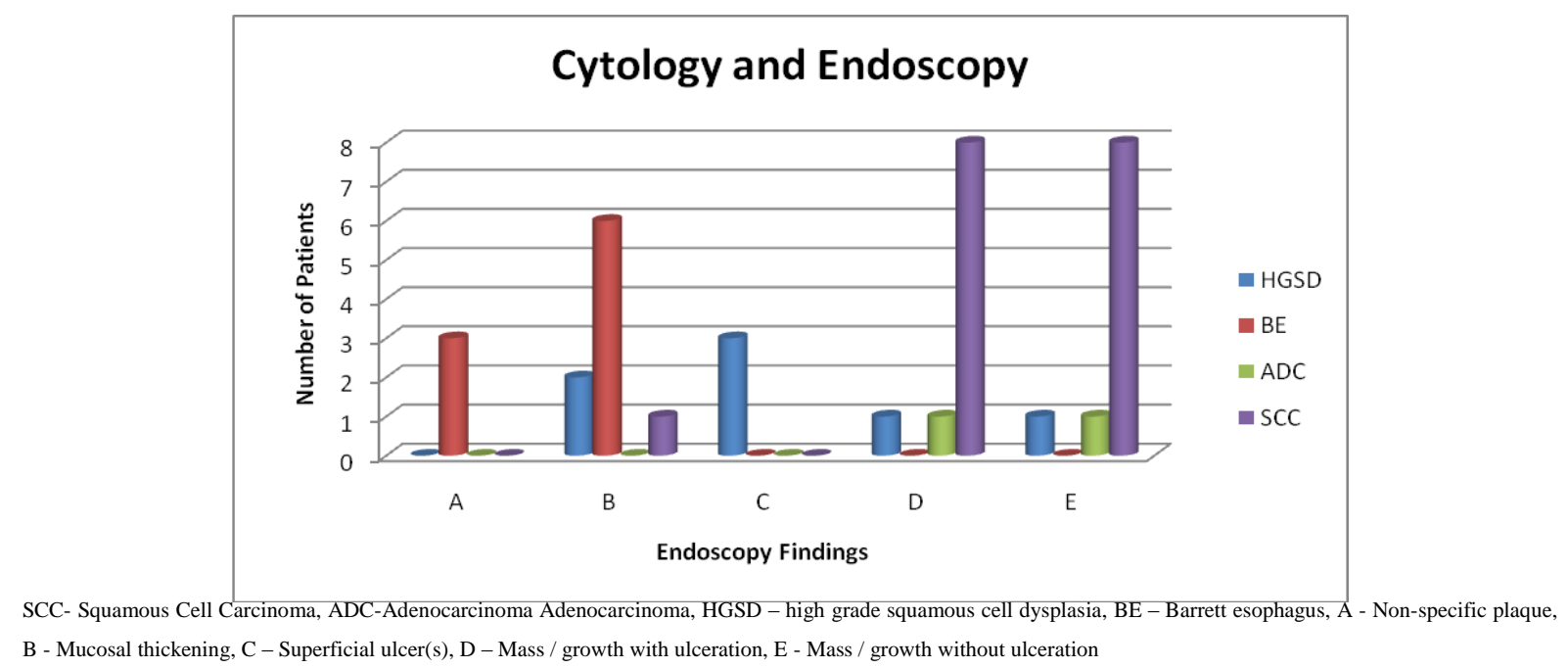

Fig. 2: Cytology and Endoscopy findings of premalignant and malignant esophageal lesions.

Endoscopic finding of salmon colored nonspecific plaque and/or mucosal thickening represented BE (9/9 patients), whereas mucosal thickening and superficial ulcers showed HGSD (5/7 patients). Endoscopic mass with or without ulcer were usually malignant lesions, either SCC (8/9 patients) or ADC (2/2 patients). The ratio of SCC to ADC of esophagus was 8.5: 1. Thus SCC was more common than ADC (Fig. 2).

\section{DISCUSSION:}

Esophageal cancer is an aggressive disease with a poor prognosis. The main reason for this poor prognosis is that most cases are asymptomatic and go undetected until they have spread beyond the esophagus and are unresectable. In this setting, successful strategies for primary prevention and early detection of curable lesions are critically needed to control this disease.

Esophageal malignancy most likely develops through a dysplasia-neoplasia sequence similar to other forms of cancer [6]. HGSD and BE are the two established premalignant conditions of SCC and ADC respectively. This implies that there are microscopic changes, such as nuclear enlargement and clumping of chromatin, that are present before the development of endoscopically visible lesions [7]. These premalignant changes can be easily picked up on endoscopic brushing cytology under direct vision.

\subsection{Barrette's esophagus:}

Most adenocarcinoma cases arise in the setting of a detectable preneoplastic lesion known as Barrett's esophagus (BE). It has become well established that gastroesophageal reflux disease (GERD) is a common and strong risk factor [8].

Recently there has been an increase in incidence of esophageal ADC in western countries in patients with BE. This occurs in a well characterized sequence. In reaction to chronic gastrointestinal reflux, columnar metaplasia of the normal stratified squamous epithelium of distal esophagus occurs, resulting in Barrett's epithelium (Fig.3). Further genetic alterations in this epithelium lead to dysplasia and ultimately to adenocarcinoma. This subgroup with BE may benefit from regular surveillance to identify progression to dysplasia prior to the development of ADC.

In the present study, we found nine cases of BE which appeared as salmon colored patch and mucosal thickening on endoscopy. Szántó I et al [9] also described salmon colored mucosal thickening as diagnostic of Barrett's esophagus. The normal esophageal lining (squamous mucosa) is light pink or white. BE was seen in female in fourth decade while ADC in male sixth decade. This gives a lead period of almost two decades.

Endoscopic surveillance for patients with BE is recommended to identify curable neoplasia and is based on a number of assumptions: (a) In the absence of surveillance, patients with BE have decreased survival because of deaths from esophageal adenocarcinoma; (b) surveillance of patients with BE reliably detects curable neoplasia (dysplasia or early cancer); and (c) treatment of esophageal neoplasia detected by surveillance prolongs survival [5].

Not all cases of Barrett's esophagus land up in adenocarcinoma. The risk factors associated with development of adenocarcinoma in Barrett's esophagus are: elderly male, long segment Barrett's $(>3 \mathrm{~cm})$, specialized columnar epithelium, high grade dysplasia. This high risk group needs surveillance with the goal of identification of epithelial dysplasia before adenocarcinoma has intervened [10] Thus, the patients of Barrett's esophagus are advised follow up. 


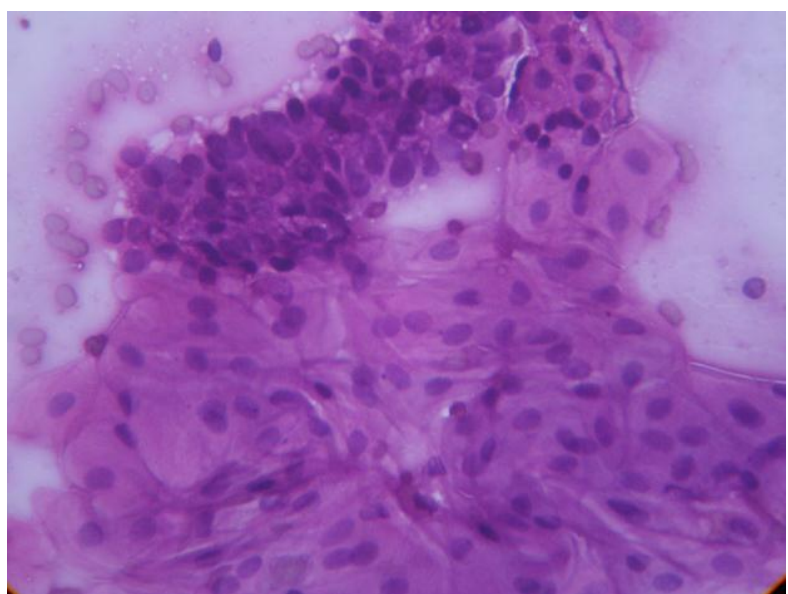

Fig. 3: Photomicrograph of Barrette's Esophagus showing squamous cells and metaplastic columnar cells. $\mathbf{H} \& \mathbf{E}(100 \mathrm{X})$

\subsection{High grade squamous cell dysplasia:}

Dysplasia is a disordered growth of epithelium that can be regarded as precancerous, and includes intraepithelial lesions that are not sufficiently atypical to designate as carcinoma in situ. Epithelial dysplasia is characterized by cellular atypia, abnormal differentiation and disorganized architecture, all of these features being more prominent in severe or high-grade dysplasia (Fig.4).

In the present study HGSD was more common in male in the fifth decade while SCC was more common in male in $7^{\text {th }}$ decade. This lead period of one decade can be used for the early diagnosis and treatment of SCC. High-grade lesions revealed nuclear enlargement and hyperchromasia increased nuclear-cytoplasmic ratio and coarse chromatin, a finding similar to the description in study by Roth M.J. et al [11]. These changes were seen mostly in the intermediate and superficial squamous cells. Nuclei were enlarged up to 3 times the size of normal cells [12].

The distinction between repair atypia and carcinoma in esophageal brushings is often difficult. The most reliable criteria for malignancy included sharply angulated nuclear rims, a high nuclear/cytoplasmic ratio, and coarsely granular chromatin.

Nuclear pleomorphism is a more consistent feature, but it showed considerable overlap with epithelial repair. Cytological features of multiple nucleoli, macronucleoli, and loss of polarity were common to both repair and carcinoma [13].

Squamous epithelial dysplasia is frequently found in the esophagus with squamous cell carcinoma, and several studies have also suggested its significance as a precancerous lesion [14].

In the present study, out of the seven cases of high grade dysplasia, two cases turned out to be of squamous cell carcinoma on biopsy. The reason for false negative diagnosis in the present study was nonsampling of malignant cells and underestimation of malignancy as high grade dysplasia. Similar reason for the false negative diagnosis of squamous cell carcinoma as high grade dysplasia was cited by Hishon S et al [15], Chamber LA and Clark II WE [16], Geisinger KR [17]. Thus, the patients with HGD should be advised biopsy follow up to rule out malignancy.

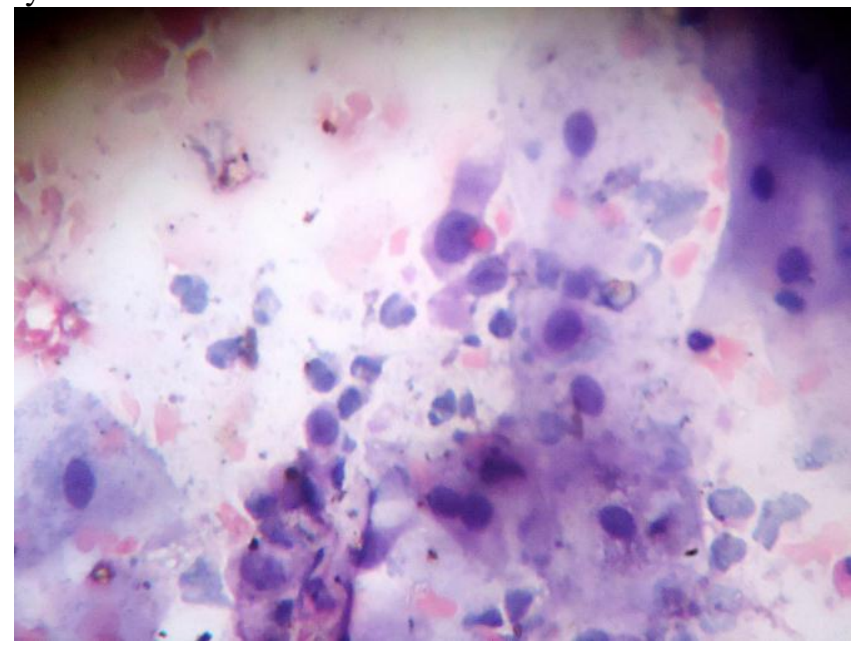

Fig. 4: Photomicrograph of high grade squamous cell dysplasia. H \& E (100 X) 


\subsection{Squamous Cell Carcinoma:}

In India, squamous cell carcinoma has remained the most common esophageal malignancy between 1989 and 2004. It is unlikely that it will be surpassed by adenocarcinoma of esophagus in the near future. This is in contrast to reports of an increasing incidence of adenocarcinoma from developed countries [18, 19].

Kobayashi S and Kasugai T [20] had classified lesions endoscopically as mucosal elevations, thick folds, mass with ulceration, mass without ulceration, etc. They reported the diagnosis of malignancy in these lesions by cytology to the value of 71.4\%. Shroff CP and Nanivadekar SA [21] found that masses with ulceration are frequently malignant. The positivity of reporting malignancy in this type by endoscopic cytology was superior to endoscopic biopsy. The observations of present study are in agreement with the above studies, masses with ulceration were diagnosed as malignant by cytology.

The prominent cytomorphological features of squamous cell carcinoma found in present study were cytoplasmic keratinization with nucleus showing features of malignancy like round to polygonal cells to bizarre cells, fiber cells or tadpole cells having abundant cytoplasm which stains eosinophillic in $\mathrm{H}$ \& $\mathrm{E}$ stains, and red or orange by Papanicolaou's stain. The nuclear: cytoplasmic ratio is increased and the nucleus contains deep staining chromatin [22]. Anisonucleosis is seen. The smear background may be hemorrhagic or dirty. Similar cytomorphological features were suggested by Howell L.P. et al [23] and Roth M.J. et al [11].

In the recent three decades, adenocarcinoma has surpassed squamous cell carcinoma of esophagus in developed countries $[18,24]$. Our findings showed that SCC was the most frequent esophageal tumor (17 cases) followed by ADC (two cases). Thus the frequency of SCC and ADC in our study was $89.5 \%$ and $10.5 \%$. It was reported by Cherian et al [18] as $92 \% \& 8 \%$ respectively.

The occurrence of the multiple independent squamous cell carcinoma of the esophagus is not rare. Taking multiple biopsies of esophagus, at different levels is practically not possible. Endoscopic brush cytology can be very helpful in such situation because of its ability to sample a greater area of the esophagus $[14,25,26]$.

\subsection{Adenocarcinoma:}

There has been an increase in the incidence of adenocarcinoma of the esophagus in developed countries. However, in India, squamous cell carcinoma still remains the most common esophageal malignancy [18, 19]. The prognosis of patients with adenocarcinoma of esophagus is poor: the majority of patients diagnosed with adenocarcinoma die within 12 months [27].

According to the study conducted by Cherian JV et al [18], the mean age of patients with adenocarcinoma has been decreasing.

Table 2: Mean age of patients with esophageal adenocarcinoma; Cherian JV et al [18].

\begin{tabular}{|l|l|l|l|l|}
\hline Years & $\mathbf{1 9 8 9 - 9 2}$ & $\mathbf{1 9 9 3 - 9 6}$ & $\mathbf{1 9 9 7 - 2 0 0 0}$ & $\mathbf{2 0 0 1 - 0 4}$ \\
\hline Mean age & $54.3 \pm 16.0$ & $58.0 \pm 8.7$ & $55.6 \pm 10.2$ & $49.3 \pm 13.5$ \\
\hline
\end{tabular}

In the present study the mean age of presentation was 48 yrs while that of SCC was 57.7 yrs. Thus, according to the present study, ADC presented a decade earlier than squamous cell carcinoma.

Endoscopically, adenocarcinoma presented with mass with or without ulcer in the distal third of the esophagus. None involved the middle or upper third of the esophagus [18].

Microscopically, malignant cells of adenocarcinoma were arranged in loosely cohesive sheets and clusters as well as isolated malignant cells. The cell clusters have frayed irregular margins and haphazard array of crowded and overlapping abnormal nuclei indicating loss of polarity. The nuclei are hyperchromatic, pleomorphic, with irregular nuclear membranes and prominent nucleoli. Cells display delicate cytoplasm. Smear background is dirty indicating tumor diathesis and necrosis [28].

\section{CONCLUSION:}

The endoscopic brushing cytology under direct vision has several procedural advantage of obtaining the adequate diagnostic material from the selective sites, multiple sampling, repeatability of brushing, no additional discomfort or risk associated with the procedure, minimal invasiveness and rapid turnover time. It is more useful when the lesions are large or are multiple; when patients refuse biopsy since patient compatibility for cytology is better than that for biopsy. Unnecessary biopsies can be avoided as only those patients in whom premalignant and malignant lesions are found on cytology can undergo biopsy.

SCC is still the most common esophageal malignancy. Adenocarcinoma presented a decade earlier than squamous cell carcinoma. However, the premalignant lesions presented a decade or two earlier than the malignant lesions. Screening and surveillance by using endoscopic brush cytology during this lead period can drastically alter the outcome in terms of morbidity and mortality. 
Thus to conclude, endoscopic brushing cytology is the best procedure for screening of patients considered high risk for squamous cell carcinoma and for surveillance of Barrett's esophagus.

\section{REFERENCES}

[1]. Flejou J F. Berrett's esophagus: from metaplasia to dysplasia and cancer. Gut 2005; 54(Suppl I): i6-i12.

[2]. Kato H, Tachimori Y, Watanube H, Yamaguchi H, Ishikawa J, Itabashi M. Superficial esophageal carcinoma. Surgical treatment and results. Cancer. 1990; 66: 2319-23.

[3]. Hirschowitz BI. A fibre optic flexible oesophagoscope. Lancet 1963; 2: 388

[4]. Karmarkar P, Wilkinson A, Manohar T, Joshi A, Mahore S. Diagnostic utility of endoscopic brush cytology in upper gastrointestinal lesions and its correlation with biopsy. IOSR-JDMS. 2013 Mar-Apr; 5 (2): 32-36.

[5]. Sharma P, Mcquaid K, Dent J, Fennerty MB, Sampliner R, Spechler S, et al. A Critical Review of the Diagnosis and Management of Barrett's Esophagus: The AGA Chicago Workshop Gastroenterology 2004;127:310-330.

[6]. Stoner GD, Gupta A. Etiology and chemoprevention of esophageal squamous cell carcinoma. Carcinogenesis 2001; 22:17371746.

[7]. Jacobson BC. Endoscopic Screening for Squamous Cell Carcinoma of the Esophagus. Faigel DO, Kochman ML. Endoscopic Oncology: Gastrointestinal Endoscopy and Cancer Management. Humana Press, Totowa, NJ. 2006 :9-15

[8]. El-Serag H B. Lagergren J. Alcohol Drinking and the Risk of Barrett's Esophagus and Esophageal Adenocarcinoma .Gastroenterology. 2009 April; 136(4): 1155.

[9]. Szántó I, Banai J, Vámosi-Nagy I, Nagy P, Bajtai A. Significance of endoscopic biopsy and cytology in the diagnosis of esophageal squamous cell carcinoma. Magy Onkol. 2006; 50(1):39-41.

[10]. Goldblum JR. Barrett's esophagus and Barrett's-related dysplasia. Mod Pathol 2003; 16(4):316-324

[11]. Roth M J, Liu S, Dawsey S M, Zhou B, Copeland C, Wang G, Soloman D, Baker S, Giffen C A, Taylor P R. Cytologic detection of esophageal squamous cell carcinoma and precursor lesions using balloon and sponge samplers in a symptomatic adults in Linxian, China. Cancer 80, 1997: 2047-59.

[12]. Prolla JC, Reilly W, Kirsner JB, Cockerham L. Direct-vision endoscopic cytology and biopsy in the diagnosis of esophageal and gastric tumors: current experience. Acta Cytol. 1977 May-Jun; 21(3): 399-02.

[13]. Hoover L, Berman J J. Diagnostic Cytopathology Epithelial repair versus carcinoma in esophageal brush cytology. 02/1988; 4(3):217-23

[14]. Takubo K, Tsuchiya S, Fukushi K, Shirota A, Mitomo Y. Dysplasia and reserve cell hyperplasia-like change in human esophagus. Acta Pathol Jpn 1981; 31: 999-1013.

[15]. Hishon S, Lovell D, Gummer JWP, Smithies A, Shawdon H, Blendis LM. Cytology in the diagnosis of oesophageal cancer. Lancet 1976; 1: 296-97.

[16]. Chambers LA, Clark II WE. The endoscopic diagnosis of gastroesophageal maliganancy. Acta Cytol 1986; 30: 110-14.

[17]. Geisinger KR. Endoscopic biopsies and cytologic brushings of the esophagus are diagnostically complementary. Am J Clin Pathol. 1995; 103: 295-99.

[18]. Cherian JV, Sivaraman R, Muthusamy AK, Jayanthi V. Carcinoma of the esophagus in Tamil Nadu (South India): 16-year trends from a tertiary center. J Gastrointestin Liver Dis. 2007 Sep; 16(3): 245-49.

[19]. Devesa SS, Blot WJ, Fraumeni JF. Changing patterns in the incidence of esophageal and gastric carcinoma in the United States. Cancer 1998; 83: 2049-2053.

[20]. Kobayashi S, Kasugai T. Brushing cytology for the diagnosis of gastric cancer involving the cardia or the lower esophagus. Acta Cytol. 1978; 22: 155-57.

[21]. Shroff CP, Nanivadekar SA. Endoscopic brushing cytology and biopsy in the diagnosis of upper gastrointestinal tract lesions. A study of 350 cases. Acta Cytol. 1988 Jul-Aug; 32 (4): 455-60.

[22]. Vidyavathi K, Harendrakumar ML, Lakshmana Kumar YC. Correlation of endoscopic brush cytology with biopsy in diagnosis of upper gastrointestinal neoplasms. Indian J Pathol microbial. 2008 Oct-Dec; 51(4):489-92.

[23]. Howell LP, Wright AL, Calafati SA, Rosen S, Koprowska I. Cytodiagnosis of in situ and early carcinoma of the upper gastrointestinal tract. Acta Cytol. 1985 May-Jun; 29 (3): 269-73.

[24]. Weiderpass E, Pukkala E. Time trends in socioeconomic differences in incidence rates of cancers of gastro-intestinal tract in Finland. BMC Gastroenterol. 2006 Dec; 6:41.

[25]. Kuwano H, Nakajima M, Miyazaki T, Kato H. Distinctive clinicopathological characteristics in esophageal squamous cell carcinoma. Ann Thorac Cardiovasc Surg. 2003 Feb; 9(1): 6-13.

[26]. Sugimachi K, Sumiyoshi K, Nozoe T, et al. Carcinogenesis and histogenesis of esophageal carcinoma. Cancer 1995; 75: 1440-5.

[27]. SJ Lowry. Modifiable risk factors for esophageal adenocarcinoma in Barrett's esophagus Feb. 17, 2013.

[28]. Bergman S and Geisinger K R. Alimentary Tract. In: Bibbo M, Wilber DC (ed). Comprehensive Cytopathology. 3rd Edn. Philadelphia. W B Saunders. 2008: 373-85. 